\title{
Further development of the kinematic and aerodynamic modeling and analysis of flapping wing ornithopter from basic principles
}

\begin{abstract}
The basis of this work was to understand the generation of lift and thrust of a flapping biwing ornithopter, which is influenced by its geometrical, dynamic, kinematic and aerodynamic features by following a generic approach in order to identify and mimic the mechanisms. As further development of earlier work, three-dimensional rigid thin wing is considered in flapping and pitching motion using strip theory and two-dimensional unsteady aerodynamics for idealized wing in pitching and flapping oscillations with phase lag. Later, parametric study is carried out to attain a complete cycleôs lift and thrust physical characteristics for evaluating the plausibility of the aerodynamic model and for the synthesis of an ornithopter model with simplified mechanism. Further investigation is conducted to identify individual contribution of generic motion towards the flight forces. Results are assessed in comparison with existing theoretical and experimental results as appropriate.
\end{abstract}

Keyword: Bi-wing-ornithopter; Flapping wing aerodynamics; Ornithopter; Unsteady aerodynamics 